The Astrophysical Journal, submitted July 11, accepted OCtober 12

Preprint typeset using $\mathrm{LAT}_{\mathrm{E} X}$ style emulateapj v. 14/09/00

\title{
BLACK HOLES OF ACTIVE AND QUIESCENT GALAXIES: I. THE BLACK HOLE-BULGE RELATION REVISITED
}

\author{
AMRI WANDEL \\ Racah Inst. of Physics, The Hebrew University, Jerusalem 91405,Israel \\ amri@vms.huji.ac.il \\ The Astrophysical Journal, submitted July 11, accepted October 12
}

\begin{abstract}
Massive Black Holes detected in the centers of many nearby galaxies show an approximately linear relation with the luminosity of the host bulge, with the black hole mass being 0.001-0.002 of the bulge mass. Previous work suggested that black holes of active (Seyfert 1) galaxies follow a similar relation, but apparently with a significantly lower value of $M_{\mathrm{BH}} / M_{\text {bulge }}$ (Wandel 1999). New data show that this difference was mainly due to over-estimating the black hole mass in quiescent galaxies and over-estimating the bulge magnitude of Seyfert galaxies. Using new and updated data we show that AGNs (Seyfert galaxies and quasars) follow the same BH-bulge relation as ordinary (inactive) galaxies. We derive the $\mathrm{BH} /$ bulge relation for a sample of 55 AGNs and 35 quiescent galaxies, finding that broad line AGNs have an average black hole/bulge mass fraction of $\sim 0.0015$ with a strong correlation $\left(M_{\mathrm{BH}} \propto L_{\mathrm{bulge}}^{0.9 \pm 0.16}\right)$. This BH-bulge relation is consistent with the BH-bulge relation of quiescent galaxies and much tighter than previous results. Narrow line AGNs appear to have a lower ratio, $M_{\mathrm{BH}} / M_{\text {bulge }} \sim 10^{-4}-10^{-3}$. We find this to be a more general feature, the $\mathrm{BH} /$ bulge ratio in AGNs being inversely correlated with the emission-line width, implying a strong linear relation between the size of the broad emission line region and the luminosity of the bulge. Finally, combining AGNs with observed and estimated stellar velocity dispersion, we find a significant correlation $\left(M_{\mathrm{BH}} \propto \sigma^{3.5-5}\right)$, consistent with that of quiescent galaxies.
\end{abstract}

Subject headings: black hole physics, galaxies:bulges, galaxies:nuclei, galaxies:active, galaxies: Seyfert, quasars:general

\section{INTRODUCTION}

Compact dark masses, probably massive black holes (MBHs), have been detected in the cores of many nearby galaxies (Kormendy and Richstone 1995, Kormendy \& Gebhardt (2001)). Magorrian et al. (1998) presented a sample of 32 nearby galaxies with MBHs and suggested that the black hole $(\mathrm{BH})$ mass is proportional to the luminosity of the host galaxy or host bulge (or equivalently, to their mass) hereafter referred to as the $\mathrm{BH} /$ bulge relation, with the $\mathrm{BH}$ mass being about 0.006 of the mass of the spheroidal bulge (although with a significant scatter).

In addition to the MBHs detected by techniques of stellar and gas kinematics, the masses of about three dozen $\mathrm{MBHs}$ in AGNs have been estimated by reverberation mapping of the broad emission-line region. High quality reverberation data and virial $\mathrm{BH}$ mass estimates are presently available for 20 Seyfert 1 nuclei (Wandel, Peterson and Malkan 1999, hereafter WPM) and 17 PG quasars (Kaspi et al. 2000). Laor (1998) suggested that the masses of BHs in quasars (estimated empirically) and their host bulges follow the $\mathrm{BH} /$ bulge relation of Magorrian et al. (1998). Using the WPM Seyfert sample with bulge estimates from Whittle (1992) Wandel (1999) found that the $\mathrm{BH} /$ bulge ratio is significantly lower (on average, by a factor of $\sim 20$ ) than the ratio found by Magorrian et al. (1998) for $\mathrm{MBHs}$ in quiescent galaxies.

Recent research using higher quality HST data and a more careful treatment of the modeling uncertainties give lower values for black hole masses in nearby galaxies (Merritt and Ferrarese 2001a), with an average black hole to bulge mass ratio of $\sim 0.001$ and a nearly linear BH-bulge relation $M_{\mathrm{BH}} \propto L_{\text {bulge }}^{1.1}$ (Kormendy \& Gebhardt 2001).

The updated lower dynamical BH mass estimates for quiescent galaxies reduced the discrepancy between inactive galaxies and reverberation mapped Seyferts, but Consequently, the quasars in Laor's (1998) sample appear to have larger $\mathrm{BH}$-bulge ratios than inactive galaxies and of less luminous AGNs. To explain this Laor (2001) suggested a nonlinear BH-bulge mass relation, $M_{\mathrm{BH}} \sim M_{\text {bulge }}^{1.5}$.

Ferrarese and Merritt (2000) and Gebhardt et al. (2000a) have found that the mass of MBHs in quiescent galaxies is better correlated with the stellar velocity dispersion in the bulge than with the bulge luminosity. Although to date there are only a few (less than ten) Seyfert galaxies with reliable measurements of stellar velocity and with reverberation BH mass estimates (Ferrarese et al. 2001), they seem to be consistent with the BH-velocity dispersion relation of inactive galaxies.

Recently McLure \& Dunlop (2001, hereafter MD01) have studied the BH-bulge relation of 30 quasars, combined with the Seyfert galaxies in the WPM sample for which they estimate bulge magnitudes from HST imaging and bulge-disk decomposition. Assuming a flattened BLR geometry and a biased distribution of inclinations MD01 suggest that the actual $\mathrm{BH}$ mass of AGNs is 3 times larger than inferred from the reverberation virial method assuming isotropic geometry. While at present a factor of 2-3 in the $\mathrm{BH}$ reverberation mass cannot be ruled out, systematically increasing the Seyfert $\mathrm{BH}$ masses by a factor of 3 seems to violate the agreement between Seyferts and quiescent galaxies in the $\mathrm{BH}$-velocity dispersion relation (fig. 2 of Ferrarese et al. 2001). 
In this work we reconcile the $\mathrm{BH}$-bulge mass relation of AGN with that of quiescent galaxies, analyzing the factors which led to the earlier apparent discrepancies between Seyferts, quasars and inactive galaxies, and demonstrate that all three groups do follow the same BH-bulge mass relation without a spatial geometry or bias for AGNs. In section 2 we present the sample, including 23 Seyfert galaxies, 32 quasars and 35 inactive galaxies with reliable $M_{\mathrm{BH}}$ and bulge data. In section 3 we show that the $\mathrm{BH}-$ bulge mass relation of the combined Seyfert+quasar sample is consistent with that of inactive galaxies, both in slope and in normalization, discuss the uncertainties and point out the factors that produced the apparent discrepancy in the $M_{\mathrm{BH}}-M_{\text {bulge }}$ relation between Seyferts and inactive galaxies in previous works. We also find that Narrow Line Seyfert 1s and narrow line quasars have significantly lower $M_{\mathrm{BH}} / M_{\text {bulge }}$ ratios than broad line AGNs and inactive galaxies, which is the result of a systematic trend (section 4) - a strong correlation between the BH mass to bulge luminosity (or mass) ratio and the emission line width of the active nucleus. In section 5 we analyse the implications of the $\mathrm{BH}$ mass - stellar velocity dispersion relation, while section 6 discusses possible reasons for the lower $\mathrm{BH} /$ bulge ratio of narrow line AGNs.

\section{DATA}

We compile a sample of 55 AGNs with $\mathrm{BH}$ mass and bulge magnitude. The AGN data are listed in tables 1 (Seyferts) and 2 (quasars). We use $H_{o}=80 \mathrm{~km} \mathrm{~s}^{-1} \mathrm{Mpc}^{-1}$ and $q_{0}=0.5$. We convert magnitudes from other works to the $\mathrm{V}$ band, using the relations $V-R=0.8$ and $B-V=$ 0.8 . We express the bulge magnitude in units of $L_{\odot}$ using the standard relation $L_{\text {bulge }} / L_{\odot}=0.4\left(-M_{V}+4.83\right)$. To find the bulge mass we use the relation $M / M_{\odot}=$ $1.18\left(L / L_{\odot}\right)-1.11$ (Magorrian et al. 1998).

\subsection{Seyferts}

We use the sample of 17 Seyferts in Wandel, Peterson and Malkan (1999), supplemented by 6 more Seyferts with reliable $\mathrm{BH}$ mass or bulge magnitude data: MRK 279 (Santos Lleo et al. 2001), NGC 3516 and NGC 4593 (Ho 1999, Gebhardt et al. 2000b) and MRK 841, NGC 4253 and NGC 6814 (bulge magnitudes from Virani et al. , BH mass from L-R relation (eq. 2 below, cf. Laor 2001). For the Seyfert galaxies in the WPM sample we use the bulge magnitude given by MD01, estimated from HST imaging and two dimensional bulge-disk decomposition. This method is more accurate than the empirical formula for the bulge/total ratio depending on the Hubble type (Simien \& deVaucouleurs 1986; Whittle et al. 1992) used in Wandel (1999). The bulge luminosity obtained by bulge/disk decomposition of galaxy images is systematically lower than the empirical estimate: for the 15 Seyferts common to Wandel (1999) and MD01 the latter have an average bulge magnitude lower by a factor of 3 . However, the difference in the bulge magnitude estimated by the two methods is not uniform: for individual objects it varies between 1 and 10. We find that the correction factor (that is, the ratio of the bulge luminosity derived by MD01 from HST imaging and that estimated from the Simien \& deVaucouleurs (SdV) relation) is correlated with the FWHM of the broad emission lines (fig. 1). For example, while the
Seyferts with the broadest lines (F9, IC 4329A, NGC 3227 and NGC 4151) have correction factors of order 10, the narrow line objects (MRK 335 and the marginally narrow line $3 \mathrm{C} 120$ ) have a negligible correction, $\approx 1$. The best fit to the bulge luminosity correction for the 15 Seyferts in MD01 is

$$
\log \left(L_{\mathrm{SdV}} / L_{\mathrm{img}}\right)=1.3 \log \left(\frac{\operatorname{FWHM}(\mathrm{H} \beta)}{10^{3} \mathrm{~km} \mathrm{~s}^{-1}}\right)-0.17
$$

The correlation coefficient is $\mathrm{R}=0.77$ with a $<0.1 \%$ probability of chance correlation. This empirical correlation is so tight that we use it to predict the actual bulge magnitude from the SdV relation for Seyferts which do not have directly measured bulge luminosity. With the empirical formula we obtain bulge magnitudes for the five Seyferts in our sample which do not have measured bulge luminosity (AKN 120, MRK 110, MRK 279, NGC 3516, NGC 4593). In the above analyses we have used for 3C390.3 a FWHM $\mathrm{H} \beta=3500 \mathrm{~km} \mathrm{~s}^{-1}$ (Crenshaw 1986) which is substantially lower than the value of $10^{4} \mathrm{~km} \mathrm{~s}^{-1}$ reported in WPM; this very broad feature may be temporary, due to the line profile variability (Peterson, private communication).

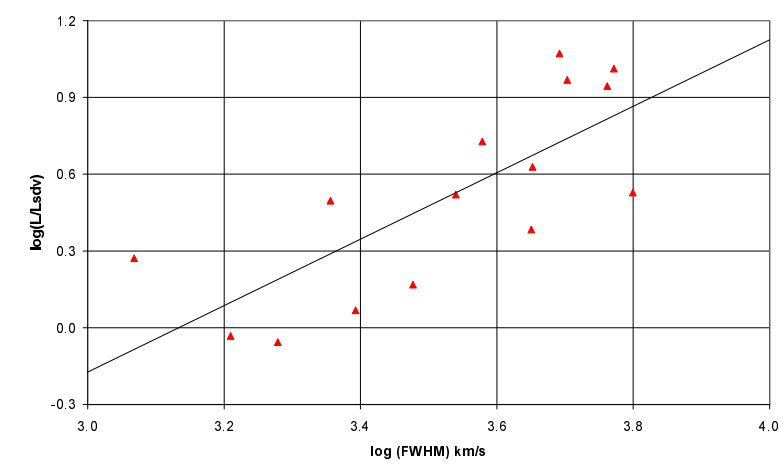

FIG. 1.- The bulge luminosity correction: the difference between the bulge/disc decomposition method (MD01) and the empirical Simien-de Vaucouleurs method (bulge/total dependence on Hubble type) vs. the $\mathrm{H} \beta$ line width for Seyfert 1 galaxies.

\subsection{Quasars}

We adopt the quasar sample of MD01, supplemented by PG1425+267 (Kirhakos et al. 1999) and PG1704+60 (BH mass from Kaspi et al. 2000, host magnitude from Hamilton, Casetano and Turnshek 2000).

The virial formula for the $\mathrm{BH}$ mass in AGNs requires the size and gas velocity of the broad emission line region. Most of the quasars do not have reverberation data. For those we estimate the size of the BLR using the empirical correlation

$$
R_{B L R}=33\left(\nu L_{\nu}(5100) / 10^{44} \mathrm{erg} \mathrm{s}^{-1}\right)^{0.7} \text { light }- \text { days }
$$

between the BLR size and the continuum luminosity (Kaspi et al. 2000). We test the goodness of the empirical R-L relation for the quasars with reverberation data. There are four quasars in the MD01 sample with reverberation data (Kaspi et al. 2000) - PG0052, PG0953, 3C273 and PG1307. Comparing the $\mathrm{BH}$ masses the agreement 


\begin{tabular}{|c|c|c|c|c|c|c|c|c|}
\hline $\begin{array}{l}\text { Name } \\
(1)\end{array}$ & $\begin{array}{r}\text { FWHM } \\
(2)\end{array}$ & $\begin{array}{r}\log L_{V, T} \\
(3)\end{array}$ & $\begin{array}{r}\log L_{V, i m g} \\
(4)\end{array}$ & $\begin{array}{r}\log \left(M_{\mathrm{bul}}\right) \\
(5)\end{array}$ & $\begin{array}{r}\log \left(M_{\mathrm{BH}}\right) \\
(6)\end{array}$ & $\begin{array}{r}\log \left(\frac{M_{\mathrm{BH}}}{M_{b u l}}\right) \\
(7)\end{array}$ & $\begin{array}{r}\sigma\left(\mathrm{km} \mathrm{s}^{-1}\right) \\
(8)\end{array}$ & $\begin{array}{r}\delta \log L_{b u l} \\
(9)\end{array}$ \\
\hline $3 \mathrm{C} 120$ & 2.21 & $9.97^{\mathrm{a}}$ & 10.03 & 10.72 & 7.49 & -3.23 & 162 & 0.52 \\
\hline 3 C 390.3 & 10.5 & 10.70 & 10.18 & 10.90 & 8.59 & -2.31 & $\ldots$ & 0.52 \\
\hline A kn 120 & 5.85 & 10.28 & $9.45^{c}$ & 10.04 & 8.29 & -2.65 & $\ldots$ & $\ldots$ \\
\hline F 9 & 5.90 & 10.75 & 9.81 & 10.46 & 7.94 & -2.52 & $\ldots$ & 0.94 \\
\hline Mrk $110 *$ & 1.67 & $10.16^{\mathrm{a}}$ & $9.70^{\mathrm{c}}$ & 10.74 & 6.91 & -3.83 & 90 & $\ldots$ \\
\hline Mrk 279 & 3.41 & 10.24 & $9.72^{\mathrm{c}}$ & 10.36 & 7.41 & -2.95 & $\ldots$ & $\ldots$ \\
\hline Mrk 335* & 1.26 & $9.86^{\mathrm{a}}$ & 9.89 & 10.56 & 6.58 & -3.98 & $\ldots$ & -0.03 \\
\hline Mrk 509 & 2.86 & 10.55 & 10.06 & 10.76 & 7.98 & -2.78 & $\cdots$ & 0.50 \\
\hline Mrk 590* & 2.17 & 10.36 & 10.29 & 11.03 & 7.15 & -3.88 & 169 & 0.07 \\
\hline Mrk 817 & 4.01 & 10.32 & 9.69 & 10.33 & 7.56 & -2.77 & 140 & 0.63 \\
\hline NGC 4151 & 5.23 & 9.84 & 8.83 & 9.31 & 7.08 & -2.23 & 90 & 1.01 \\
\hline NGC 4253 & 4.10 & $\ldots$ & 9.63 & 10.25 & $7.00^{\mathrm{b}}$ & -3.25 & $\cdots$ & $\ldots$ \\
\hline NGC 4593 & 3.72 & 9.59 & $10.20^{\mathrm{c}}$ & 10.21 & $6.91^{\mathrm{b}}$ & -3.30 & 124 & $\ldots$ \\
\hline NGC 5548 & 5.50 & 10.21 & 9.68 & 10.31 & 7.83 & -2.48 & 180 & 0.53 \\
\hline NGC 6814 & 5.50 & 9.56 & 9.25 & 9.81 & $7.08^{\mathrm{b}}$ & -2.73 & $\ldots$ & 0.31 \\
\hline NGC 7469 & 3.20 & 10.21 & 10.04 & 10.74 & 6.88 & -3.86 & $\ldots$ & 0.17 \\
\hline
\end{tabular}

${ }^{a}$ Unknown Hubble type, bulge correction estimated assuming Sa

${ }^{b}$ Bulge magnitude from Virani (1999), BH mass from R-L scaling (eq. 2)

${ }^{c}$ No image, bulge luminosity estimated from FWHM- $\delta L_{\text {bulge }}$ relation (eq. 1)

Note. - Narrow line Seyferts are denoted by an asterisk. Column (2) - FWHM of (H $\beta)$, rms profile, in units of $10^{3} \mathrm{~km} \mathrm{~s}^{-1}$. (3) - absolute bulge V luminosity derived from Hubble Type empirical formula from Whittle et al. (1992), (4) - absolute bulge V luminosity derived by imaging bulge/disk decomposition from MD01, (5) - log of the galactic bulge mass $\left(M_{b u l}\right)$ in $M_{\odot}$ (MD01), (6) - black hole mass (from WPM), (7) - BH to bulge mass ratio, (8) - stellar velocity dispersion in $\mathrm{km} \mathrm{s}^{-1}$ from Nelson \& Whittle (1995) and Ferrarese et al. (2001), (9) - log bulge correction $\left(L_{V, T} / L_{\mathrm{img}}\right)$.

TABLE 2

QUASAR DATA

\begin{tabular}{|c|c|c|c|c|c|}
\hline $\begin{array}{l}\text { Name } \\
(1)\end{array}$ & $\begin{array}{l}\text { FWHM } \\
(2)\end{array}$ & $\begin{array}{l}\log L_{V} \\
(3)\end{array}$ & $\begin{array}{l}\log \left(M_{\mathrm{bul}}\right) \\
(4)\end{array}$ & $\begin{array}{l}\log \left(M_{\mathrm{BH}}\right) \\
(5)\end{array}$ & $\begin{array}{l}\log \left(\frac{M_{\mathrm{BH}}}{M_{\text {bul }}}\right) \\
(6)\end{array}$ \\
\hline $0052+25$ & 4.37 & 10.41 & 11.18 & 8.29 & -2.89 \\
\hline $0054+14$ & 9.66 & 10.65 & 11.46 & 8.91 & -2.55 \\
\hline $0137+012$ & 7.61 & 10.81 & 11.65 & 8.57 & -3.08 \\
\hline $0157+001 *$ & 2.14 & 10.93 & 11.79 & 7.70 & -4.09 \\
\hline $0204+292$ & 6.80 & 10.53 & 11.32 & 7.15 & -4.17 \\
\hline $0205+02$ & 2.90 & 9.65 & 10.28 & 7.86 & -2.42 \\
\hline $0244+194$ & 3.70 & 10.33 & 11.08 & 8.03 & -3.05 \\
\hline $0736+017$ & 2.96 & 10.65 & 11.46 & 7.99 & -3.47 \\
\hline $0923+20$ & 7.30 & 10.53 & 11.32 & 8.94 & -2.38 \\
\hline $0953+41$ & 2.96 & 10.33 & 11.08 & 8.39 & -2.69 \\
\hline $1004+13$ & 6.34 & 10.85 & 11.70 & 9.09 & -2.61 \\
\hline $1012+008$ & 2.64 & 10.77 & 11.60 & 7.79 & -3.81 \\
\hline $1020-103$ & 7.95 & 10.57 & 11.36 & 8.35 & -3.01 \\
\hline $1029-14$ & 7.50 & 10.41 & 11.18 & 9.07 & -2.11 \\
\hline $1116+215$ & 2.92 & 10.69 & 11.51 & 8.22 & -3.29 \\
\hline $1202+28$ & 5.01 & 10.33 & 11.08 & 8.29 & -2.79 \\
\hline $1217+023$ & 3.83 & 10.73 & 11.55 & 8.40 & -3.15 \\
\hline $1226+02$ & 3.52 & 10.97 & 11.84 & 8.61 & -3.23 \\
\hline $1302-10$ & 3.40 & 10.61 & 11.41 & 8.30 & -3.11 \\
\hline $1307+08$ & 5.32 & 10.37 & 11.13 & 8.05 & -3.08 \\
\hline $1309+35$ & 2.94 & 10.41 & 11.18 & 7.99 & -3.19 \\
\hline $1402+26^{*}$ & 1.91 & 9.93 & 10.61 & 7.28 & -3.33 \\
\hline $1425+267^{\mathrm{a}}$ & 9.40 & 10.57 & 11.36 & 9.32 & -2.04 \\
\hline $1444+40$ & 2.48 & 10.37 & 11.13 & 8.06 & -3.07 \\
\hline $1545+21$ & 7.03 & 10.53 & 11.32 & 8.93 & -2.39 \\
\hline $1635+119$ & 5.10 & 10.49 & 11.27 & 8.10 & -3.17 \\
\hline $1704+60^{* \mathrm{~b}}$ & 0.40 & 10.37 & 11.13 & 6.87 & -4.86 \\
\hline $2135-14$ & 5.50 & 10.61 & 11.41 & 8.94 & -2.47 \\
\hline $2141+175$ & 4.45 & 10.73 & 11.55 & 8.74 & -2.81 \\
\hline $2247+140^{*}$ & 2.22 & 10.73 & 11.55 & 7.59 & -3.96 \\
\hline $2349-01$ & 5.50 & 10.97 & 11.84 & 8.78 & -3.06 \\
\hline 2355-082 & 7.51 & 10.65 & 11.46 & 8.39 & -3.07 \\
\hline
\end{tabular}

${ }^{a}$ Kirhakos et al. 1999

${ }^{b} \mathrm{FWHM}(\mathrm{rms})$ and $M(\mathrm{rms})$ from Kaspi et al. (2000)

Note. - Narrow-line quasars are denoted by an asterisk. Column (2) - FWHM of (H $\beta)$ in units of $10^{3} \mathrm{~km} \mathrm{~s}^{-1}$. (3) - absolute bulge V luminosity (in units of $L_{\odot}$,from MD01), (4) - log of the galactic bulge mass, (5) - black hole mass, (6) - BH to bulge mass ratio 
between the empirical R-L relation and the reverberation result is within a factor of 1.5 .

There are three quasars for which our BH mass (calculated with the empirical formula) differs significantly from the values given by MD01 (after correcting for their different kinematic factor, see 2.4 below): PG1307 (the mass in MD01 is by a factor 4 too low, apparently because a mistaken FWHM), similarly for 0204+292 (3C059) MD01 get a $\mathrm{BH}$ mass too low by a factor 40 (they have FWHM=1040 $\mathrm{km} / \mathrm{s}$, while we use a $\operatorname{FWHM}(\mathrm{H} \beta)$ of $6800 \mathrm{~km} / \mathrm{s}$, cf. Erackleous \& Halpern 1994) and PG 1012+008 (their BH mass is by a factor 10 too low, probably a typo).

\subsection{Quiescent Galaxies}

For comparison with inactive or weakly active galaxies we adopt the sample of Kormendy \& Gebhardt (2001), which lists 37 galaxies with $\mathrm{BH}$ mass determinations from stellar or gas dynamics and bulge magnitudes. We use the relation $\mathrm{B}-\mathrm{V}=0.8$ in order to translate the $\mathrm{B}$ bulge magnitude to our standard $\mathrm{V}$ luminosity. We exclude the two galaxies NGC4486B and NGC5845 which have a large uncertainty (factor 10) in the $\mathrm{BH}$ mass, significantly larger than the other objects in the sample. In the $M_{\mathrm{BH}}-\sigma$ analyses we also exclude NGC 4945 which does not have a $\sigma$ observation.

\subsection{Black Hole Mass}

The BH mass of AGNs can be estimated assuming the emission-line gas is bound (see section 6.5). The virial relation the gives $M=k^{2} G^{-1} R_{b l r} v_{F W H M}^{2}$, where $k v_{F W H M}$ is the velocity dispersion in the gas (deduced from the observed width $v_{F W H M}$ of the emission lines) and the kinematic factor $k$, which depends on the kinematics, geometry and emissivity of the BLR. Assuming the velocity dispersion in the line emitting gas is isotropic we have $k=\frac{\sqrt{3}}{2}$, which gives

$$
M_{\mathrm{BH}}=1.46 M_{\odot} \times 10^{5}\left(\frac{R_{b l r}}{\mathrm{lt}-\text { days }}\right)\left(\frac{v_{F W H M}}{10^{3} \mathrm{~km} \mathrm{~s}^{-1}}\right)^{2}
$$

Non isotropic geometry and velocity dispersion (combined with a non-uniform distribution of inclinations) can give different values of the coefficient $k$ (see section 6.4). MD01 assume a flattened BLR geometry and a distribution of inclination biased to face-on, which yield $k=3 / 2$ and hence the virial $\mathrm{BH}$ mass is 3 times larger than in the isotropic geometry.

\section{THE BH-BULGE RELATION}

\subsection{The Luminosity Relation}

In fig. 2 we plot the $\mathrm{BH}$ mass vs. bulge luminosity. Notably the scatter in the Seyfert and quasar groups is quite large. We note however that all AGNs with low $M_{\mathrm{BH}} / L_{\text {bulge }}$ values are narrow emission-line objects (NLS1s or narrow line quasars). In other words, it seems that narrow line AGNs tend to have small BHs compared to their host bulges. We draw the limit between narrow and broad line Seyferts at $2200 \mathrm{~km} \mathrm{~s}^{-1}$, which is close to the traditional NLS1 definition $\left(2000 \mathrm{~km} \mathrm{~s}^{-1}\right)$ but includes a couple of narrow line objects in our sample which are very close to that definition (MRK 590, PG0157+00 and PG2247+14). The precise value is not important, as the
NLS1 definition is merely a historic convention, and our results are not very sensitive to the exact border value.

In section 4 we show that there is a strong correlation between line width and $\mathrm{BH} /$ bulge ratio, so that the transition from broad to narrow line objects is gradual, as well as the transition from high to low $\mathrm{BH} /$ bulge ratios. This correlation further supports the separation of the narrowline objects: as the $\mathrm{BH}$ mass estimate of AGNs strongly depends on the line width, removing the narrow-line objects may seem to automatically remove the outlying data points with low $\mathrm{BH} /$ bulge ratio. However, we find that this is not the case, as the $\mathrm{BH} /$ bulge mass ratio depends also on other observables: the BLR radius and the bulge magnitude. In order to test the significance of separating the narrow line objects, we compare the two correlations: $\mathrm{BH}$ mass vs. FWHM and $\mathrm{BH} /$ bulge ratio vs. FWHM. The correlation coefficients are 0.62 and 0.80 , respectively, which shows that the $\mathrm{BH} /$ bulge - FWHM relation is more significant than the mere $\mathrm{BH}$ mass dependence on the FWHM.

In section 6 we discuss a few physical and observational models which could explain such a correlation and motivate the separation of the narrow line objects. We group those objects (Narrow Line Seyfert 1s and narrow line quasars) as a separate class which we denote NLAN (Narrow Line Active Nuclei).

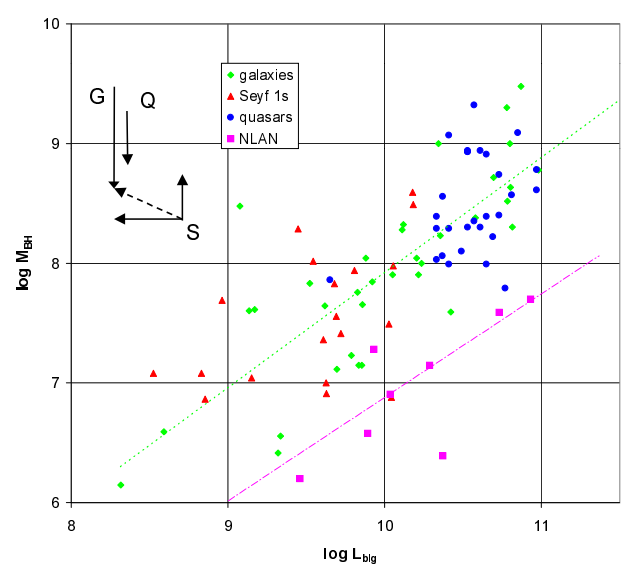

FIG. 2.- The BH mass vs. bulge luminosity. Green diamonds denote BHs detected by stellar and gas dynamics in quiescent galaxies (Kormendy \& Gebhardt 2001), blue circles - quasars (MD01), red triangles - Seyfert 1 galaxies (BH mass from WPM, bulge luminosity from MD01) and pink squares - Narrow Line Active Nuclei (NLS1s from WPM and NLQs from MD01). The least-squares linear fit for quiescent galaxies is indicated by a dashed green line and NLANs by a pink, dot dashed line. The arrows indicate the effects of the improvements in the data that cancelled the apparent former discrepancy in the $M_{B H} / L_{\text {bulge }}$ ratio between Seyferts and quiescent galaxies and quasars (see text).

The best fits to the data are ( $L$ and $M$ are in solar units)

1. Quiescent galaxies (35):

$\log M=(0.96 \pm 0.13) \log L-(1.7 \pm 1.3)$,

2. Broad line AGNs (19 Seyferts and 28 quasars)

$\log M=(0.90 \pm 0.11) \log L-(1.1 \pm 1.1)$, 
3. Narrow line AGNs (4 Seyferts and 4 quasars):

$\log M=(0.84 \pm 0.21) \log L-(1.5 \pm 2.2)$

4. Quiescent galaxies and broad line AGNs (82)

$$
\log M=(0.93 \pm 0.13) \log L-(2.9 \pm 2.6) .
$$

The fits for the $\mathrm{BH} /$ bulge mass relation, along with the corresponding correlation measures are detailed in table 3 below. The (broad line) AGNs have an almost linear fit very similar to that of inactive galaxies and to the combined sample of broad-line AGNs and quiescent galaxies. The NLAN group has a similar slope but with an $M / L$ ratio approximately a factor of 10 lower.

\subsection{The Mass Relation}

Transforming bulge luminosity to mass one finds a similar correlation between the BH mass and the bulge mass. The correlation is analogous to the $\mathrm{BH}$ mass vs. bulge luminosity relation (fig. 2), with a somewhat less steep slope (since $M_{\text {bulge }} \sim L^{1.2}$ ). Fig. 3 shows the $\mathrm{BH}$ mass vs. the bulge mass. In we list the best fits to the $M_{\mathrm{BH}}$ vs. $M_{\text {bulge }}$ relation for broad line AGNs (Seyfert 1s and quasars), narrow line AGNs (NLS1s and NL QSOs) and quiescent galaxies, as well as for the combined sample. For each subset we give the three measures of the fit: the correlation coefficient, the standard deviation of the (logarithmic) scatter, given by $d M=\left[\Sigma\left(\delta \log \left(M_{b h}\right)^{2} /(N-1)\right]^{1 / 2}\right.$ and the reduced $\chi^{2}$ of the fit. Since the BH mass estimates have systematic modeling uncertainties (section 4), we approximate the uncertainty in the $\chi^{2}$ calculation by a uniform error of 0.5 dex (factor 3 ) in the $\mathrm{BH}$ mass estimate, which is derived by combining the typical observational error with the systematic uncertainty (see section 3.3 below).

We also list the mean $\mathrm{BH} /$ bulge mass ratios and their standard deviations. In order to compare the BH-bulge correlation with that of the $M_{\mathrm{BH}}-\sigma$ relation we have added a row showing the $M_{\mathrm{BH}}-\sigma$ correlation for quiescent galaxies. Since both correlations have the same y-variable $\left(M_{\mathrm{BH}}\right)$ they can be compared using the same statitstic. For consistency we treat the errors in the BH mass for AGNs and quiescent galaxies in the same manner, although in some cases, such as the Galaxy and NGC 4258, the error in the $\mathrm{BH}$ mass is much smaller. Note that the effective error (factor 3) assumed for the BH mass is larger than the measurement uncertainty for most BHs in quiescent galaxies. Using merely the measurement uncertainty in $M_{\mathrm{BH}}$, e.g. the error bars quoted in the Kormendy and Gebhardt (2001) sample gives $\chi^{2}=3.5$ for the $M_{\mathrm{BH}}-\sigma$ relation and $\chi^{2}=10.5$ for $M_{\mathrm{BH}}$-bulge relation.

We note several important results:

1. Broad-line (BL) AGNs have a very similar distribution (for both, slope and mean $\mathrm{MH} /$ bulge ratio) to that of the quiescent galaxies.

2. In spite of their small number in out sample, removing the narrow line AGNs has a significant effect on the combined AGN sample: the total AGN sample has a significantly lower correlation coefficient, and a lower mean $\mathrm{BH} /$ bulge ratio than BL AGNs and quiescent galaxies.
3. NLANs have an average a BH/bulge ratio lower by a factor of 10 than the broad line AGN average, and the normalization of the best fit is similarly lower.

4. Quasars and Seyfert galaxies as separate groups have shallower slopes and lower correlation coefficients, due to the lower dynamical range of each of these groups separately.

5. The BH-bulge correlation of broad line AGNs is quite tight, not much less than the $M_{\mathrm{BH}}-\sigma$ relation for quiescent galaxies.

The last item is demonstrated by comparing the three correlation statistics in table 3 (the correlation coefficient, the logarithmic scatter and $\chi^{2}$ ) for the BH-bulge and the $\mathrm{BH}-\sigma$ relations. For the $47 \mathrm{BL}$ AGNs we find $0.76,0.43$ and 0.78 , respectively, nearly as tight as the $M_{\mathrm{BH}}-\sigma$ correlation of the 34 quiescent galaxies in the Kormendy and Gebhardt (2001) sample $(0.86,0.38$ and 0.60$)$. We note that excluding the Seyfert galaxy NGC 7459 (which has an exceptionally low $\mathrm{BH}$ mass relative to its luminosity) the BH-bulge correlation of the broad-line AGNs becomes even tighter $(\mathrm{dM}=0.39)$.

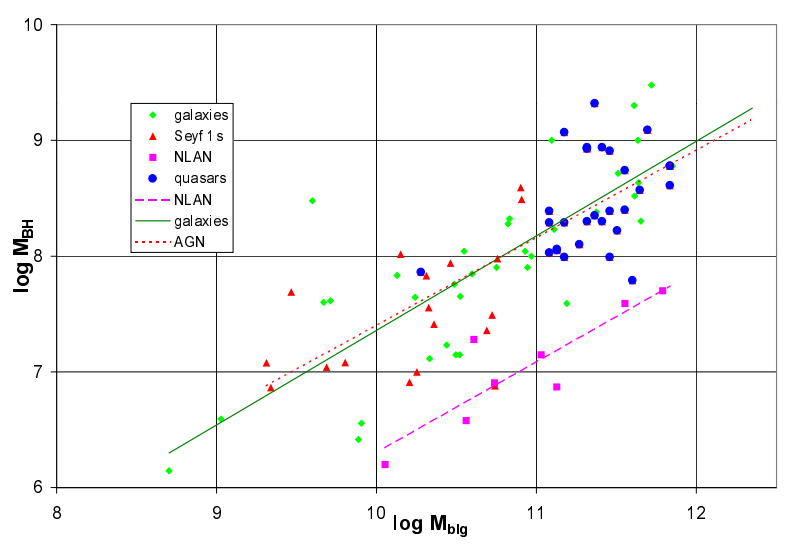

FIG. 3.- Mass estimates of MBHs plotted against the mass of the host bulge (or host galaxy for elliptical galaxies). Green Diamonds denote BHs in inactive galaxies, blue circles - quasars, red triangles - Seyfert 1 galaxies and pink squares - Narrow Line Active Nuclei (NLS1s and NLQs). The least-squares linear fits for each class are indicated by a solid green line (quiescent galaxies), dashed red line (broad line AGNs) and a pink, long dashed line (NLANs).

\subsection{Uncertainties}

The formal uncertainty in the slope of the linear fit to the (broad line) AGNs is 0.09. However, for a realistic uncertainty it is necessary to take into account the errors in the $\mathrm{BH}$ mass estimate and bulge luminosity. The errors in the $\mathrm{BH}$ mass are of two kinds: systematic errors in the reverberation mapping and virial mass calculation and measurement errors. The latter are random and typically of the order of 2-3 (see WPM for the observational uncertainty in the reverberation masses and for the error of the $L-R$ scaling method (used in our sample for most of the quasars) compared to the reverberation technique ). 
TABLE 3

BH-Bulge Mass Correlation's

\begin{tabular}{|c|c|c|c|c|c|c|c|}
\hline Set & $\begin{array}{c}\mathrm{N} \\
(1) \\
\end{array}$ & $\begin{array}{c}\mathrm{a} \\
(2) \\
\end{array}$ & $\begin{array}{c}\mathrm{b} \\
(3) \\
\end{array}$ & $\begin{array}{c}M_{\mathrm{BH}} / M_{\mathrm{blg}} \\
(4)\end{array}$ & $\begin{array}{c}\mathrm{R} \\
(5) \\
\end{array}$ & $\begin{array}{r}\delta M \\
(6) \\
\end{array}$ & $\begin{array}{l}\chi^{2} \\
(7) \\
\end{array}$ \\
\hline All AGNs & 55 & $0.74 \pm 0.11$ & $-0.09 \pm 1.22$ & $-2.93 \pm 0.57$ & 0.67 & 0.54 & 1.20 \\
\hline Broad Line AGNs & 47 & $0.73 \pm 0.09$ & $-0.15 \pm 1.02$ & $-2.80 \pm 0.48$ & 0.76 & 0.43 & 0.78 \\
\hline Quiescent Galaxies & 35 & $0.82 \pm 0.11$ & $-0.82 \pm 1.15$ & $-2.77 \pm 0.50$ & 0.80 & 0.48 & 0.96 \\
\hline BL AGNs and Q.Galaxies & 82 & $0.79 \pm 0.07$ & $-0.53 \pm 0.74$ & $-2.79 \pm 0.47$ & 0.79 & 0.45 & 0.82 \\
\hline BL quasars & 28 & $0.51 \pm 0.24$ & $2.70 \pm 2.74$ & $-2.86 \pm 0.41$ & 0.38 & 0.38 & 0.61 \\
\hline BL Seyfert 1s & 19 & $0.52 \pm 0.23$ & $2.27 \pm 2.37$ & $-2.68 \pm 0.55$ & 0.48 & 0.49 & 1.01 \\
\hline NLANs & 8 & $0.78 \pm 0.17$ & $-1.53 \pm 1.9$ & $-3.9 \pm 0.27$ & 0.88 & 0.24 & 0.27 \\
\hline Quiescent Galaxies- $\sigma$ & 34 & $3.65 \pm 0.37$ & $-0.29 \pm 0.85$ & $5.71 \pm 0.60$ & 0.86 & 0.38 & 0.60 \\
\hline
\end{tabular}

Note. - Columns: (1) N - number of objects, (2-3) coefficients of the linear fit $\log \left(M_{\mathrm{BH}}\right)=a \log \left(M_{\mathrm{bulge}}\right)+b$ and standard deviations, (4) mean $\log (\mathrm{BH} /$ bulge $)$ mass ratio and standard deviation, (5) $\mathrm{R}$ - correlation coefficient, (6) Standard deviation scatter of the fit $\delta\left(\log \left(M_{\mathrm{BH}}\right)\right)$ (7) - reduced $\chi^{2}$ assuming the error in $\log \left(M_{\mathrm{BH}}\right)$ is 0.5 dex.

The systematic errors are not random, but depend on the geometry and dynamics of the broad emission line region, and in extreme cases could amount to a factor of 3 in each direction (e.g. Krolik 2001; see section 6 below). However, since this dependence is largely unknown and can act in both directions, depending on the conditions in each AGN, we treat the systematic errors as random ones as well.

The uncertainty of a linear fit $y=a x+b$ for $\mathrm{N}$ data pairs $(x, y)$ with typical errors $\delta x$ and $\delta y$ may be estimated as $\delta a=\left[\delta a_{\text {formal }}^{2}+(a \delta x / \sqrt{N})^{2}+(\delta y / \sqrt{N})^{2}\right]^{1 / 2}$. Combining the formal error in the fit with the random measurement errors in the $\mathrm{BH}$ mass (assumed to be a factor of 3 ) and bulge luminosity (factor 2), and the systematic errors in the $\mathrm{BH}$ mass estimate (assumed to be random with a factor of 3$)$ for the broad-line AGN sample $(\mathrm{N}=47)$, gives $\delta a=0.16$, so that

$$
M_{\mathrm{BH}} \propto L_{\text {bulge }}^{0.90 \pm 0.16}
$$

and

$$
M_{\mathrm{BH}} \propto M_{\mathrm{bulge}}^{0.76 \pm 0.15}
$$

with $<M_{\mathrm{BH}} / M_{\text {bulge }}>=0.0015$.

Studying a sample of 16 AGNs Laor (2001) finds a steeper fit, $M_{\mathrm{BH}} \propto M_{\text {bulge }}^{1.53 \pm 0.14}$ (formal error only), which is $\sim 5-6 \sigma$ higher than what we find in this work $\left(M_{\mathrm{BH}} \propto\right.$ $\left.M_{\text {bulge }}^{0.73 \pm 0.09}\right)$. This difference is explained as follows. (i) Laor's fit includs quiescent galaxies, some of which have a low bulge luminosity and a low $M_{\mathrm{BH}} / M_{\text {bulge value (see sec- }}$ tion 6.2). For the AGNs separately Laor finds a less steep slope of $1.36 \pm 0.15$. Furthermore, a single point significantly influences the slope - the NLS1 NGC 4051 - which extends the dynamical range by almost an order of magnitude. If it were excluded (being a narrow line object), the slope of the fit would decrease considerably (and the standard deviation in the slope would increase). Excluding NGC 4051 and adding the measurement error would flatten the slope and increase the uncertainty in Laor's fit, which would ecome consistent with linearity.

(ii) The BH masses used by Laor $(1998 ; 2001)$ are systematically larger than those used in this work (and in MD01). The BH masses in Laor's sample are are on average larger by a factor of 1.5 than those calculated for the same quasars in our sample. This discrepancy originates in part from the different luminosity measures used: Laor used the broad band (0.1-1 micron) luminosity (Neugebauer et. 1979) while we (and MD01) use the monochromatic luminosity $\nu F_{\nu}(5100)$. The latter seems more adequate here, as we (as well as MD01) estimate the BH mass of the quasars using the empirical $R-L$ relation of Kaspi et al. (2000) which has been derived with $\nu F_{\nu}(5100)$.

We conclude that quasars, Seyfert galaxies and inactive galaxies have the same $\mathrm{BH}$-bulge relation and $\mathrm{BH} /$ bulge ratio, while NLANs seem to have $\mathrm{BH} /$ bulge ratios lower by factors of $\sim 1-30$.

\subsection{Resolving the discrepancy: Do Seyferts have a lower $\mathrm{BH} /$ bulge ratio than Quiescent Galaxies?}

Previous works have found a significant difference in the black hole mass- bulge luminosity (or mass) relationship of Seyferts and ordinary galaxies, the former showing systematically lower $M_{\mathrm{BH}} / L_{\text {bulge }}$ values (Wandel 1999 , Ho 1999, Gebhardt 2000b). The discrepancy between the $M_{\mathrm{BH}} / M_{\text {bulge }}$ ratio of quiescent galaxies and Seyferts (factor of 10-20 on average) was a result of three factors, shown schematically by the arrows in fig. 2: (i) the Magorrian $\mathrm{BH}$ masses were overestimated by an average factor of $\sim 5$ $\left(M_{\mathrm{BH}} / M_{\text {bulge }}=0.6 \%\right.$ compared with the current estimate of 0.1-0.13\% (Ho 1999; Merritt \& Ferrarese 2001a). The average decrease in the quiescent galaxy $\mathrm{BH}$ mass is shown in fig. 2 by the arrow denoted G. (ii) the Seyfert bulge luminosity calculated using the Simien de Vaucouleurs formula is too large (by a factor of $\sim 2-3$ ); the bulge lumi- 
nosity estimated by direct imaging and bulge/disk decomposition is lower by an average factor of $\sim 2(0.15$ dex in our total AGN sample, as seen in table 3, but more when applied to smaller sub-samples, e.g. to the Seyferts) represented by the horizontal arrows. (iii) Narrow Line Seyfert 1 galaxies, which do seem to have $\mathrm{BH} /$ bulge mass ratios lower than broad line active galaxies, lowered the average. Excluding the NLS1s raises the average $M_{\mathrm{BH}} / M_{\text {bulge }}$ value of AGNs by a factor of $\sim 2$, denoted in fig. 2 by the arrow pointing upwards. Items (ii-iii) combined increased the $\mathrm{BH} /$ bulge ratio of Seyferts by an average factor of $\sim 3.5$. (dashed arrow). Similarly, the disagreement between the $\mathrm{BH} /$ bulge ratios of the $\mathrm{PG}$ quasars and reverberation Seyferts (Wandel 1999) disappeared due to the revision of the Seyferts' $\mathrm{BH} /$ bulge ratio and the lower $\mathrm{BH}$ mass estimates of the quasars (the arrow denoted by $\mathrm{Q}$ in fig 2).

Given the present good agreement of AGNs and quiescent galaxies, one may ask whether there is room for systematically higher $\mathrm{BH}$ mass estimates in AGNs, e.g. due to inclination effects. In the geometry suggested by MD01, the virial BH mass of AGN would be larger on average factor of 3, compared with the mass calculated under the assumption of isotropic velocity distribution. We can check what would be the implication of such an increase on the BH-bulge and $\mathrm{BH}$-velocity dispersion relations of AGNs compared with those of quiescent galaxies (where the BH mass estimates are not affected). In order to test this hypothesis we calculate the $\chi^{2}$ of the AGN sample (including the NLANs) with the $\mathrm{BH}$ mass increased by a uniform factor 3 , with respect to the best fit to the quiescent galaxies presented in table 3 . Assuming the typical measurement + modeling error in the $\mathrm{BH}$ mass is 0.5 dex. this gives a reduced $\chi^{2}$ of 2.2 compared with 1.20 for $\mathrm{BH}$ masses calculated with an "isotropic BLR" assumption for all AGNs in our sample. A similar exercise can be done for the $\sigma-M_{B H}$ relation, which has less AGNs, but also lower spread and a smaller error in the BH mass. Increasing each BH mass by a factor of 3 , we find for the 11 Seyfert galaxies with velocity dispersion data (table 1) $\chi^{2}$ of 2.58 compared with 1.08 for the "isotropic BLR" BH masses. We conclude that the $\mathrm{BH}$ mass calculated under the "isotropic BLR" assumption for the BLR geometry is more consistent with the BH-bulge and BH-velocity dispersion relations for quiescent galaxies.

\section{THE BH-BULGE VS. EMISSION-LINE WIDTH CORRELATION}

We find that narrow-line AGNs - NLS1s and narrow line quasars - tend to have low $M_{\mathrm{BH}} / M_{\text {bulge values. This gives }}$ a physical motivation to the exclusion of the narrow line AGNs as a separate group in the $\mathrm{BH}$-bulge relation.

Mathur et al. (2001) make a similar suggestion based on a sample of 15 NLS1s, but with less reliable BH masses. Only three of the objects in their sample have black hole masses measured by reverberation , 3 more by the scaling-virial method, and the 9 remaining $\mathrm{BH}$ masses are calculated using accretion disk modeling, which is less reliable. They find that their accretion disk model gives $\mathrm{BH}$ masses too large (by a factor of 3-7) compared with the reverberation masses (where available). In order to compensate for this discrepancy they decrease the $\mathrm{BH}$ masses given by the accretion-disk model by a calibration correction factor of 5 . For the bulge magnitude Mathur et al. use Whittle (1992) with the Simien-deVaucouleurs relation (which is likely to overestimate the bulge magnitude in AGN, as discussed in above).

Considering all 55 AGNs in our sample with available $M_{\mathrm{BH}}$ and bulge magnitude we find that the smaller $\mathrm{BH} /$ bulge ratio of narrow line objects is in fact the lower end of a more general relation: the $\mathrm{BH}$ mass /bulge luminosity ratio is strongly correlated with the emissionline width.

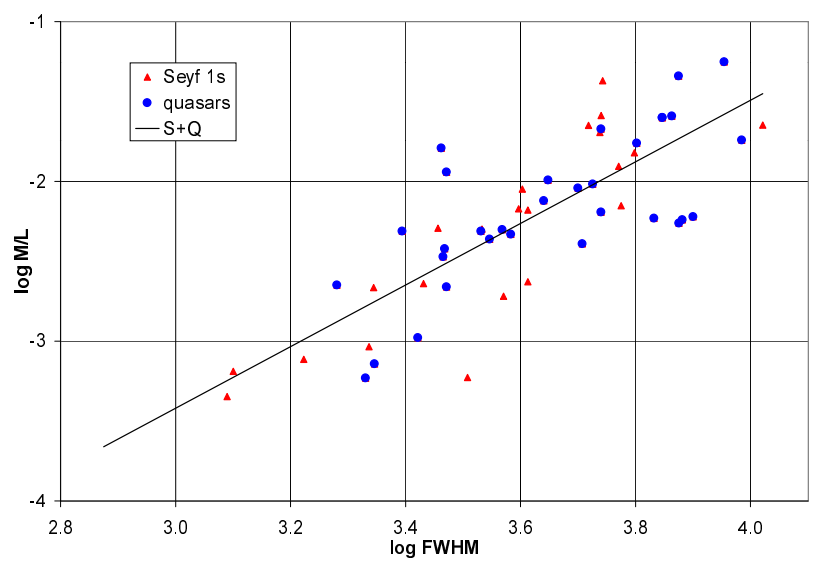

FIG. 4.- BH-to-bulge luminosity ratio plotted against the $\mathrm{H} \beta$ broad emission-line width for AGNs.Red Triangles represent Seyfert 1 galaxies and blue circles are quasars. The solid line indicates the least-squares linear fit.

In fig. 4 we plot $M_{\mathrm{BH}} / L_{\text {bulge }}$ vs. $\operatorname{FWHM}(\mathrm{H} \beta)$. The best linear least-squares fit is

$$
\log \left(\frac{M_{\mathrm{BH}}}{L_{\text {bulge }}}\right)_{\odot}=1.94 \log \left(\frac{\mathrm{FWHM}(\mathrm{H} \beta)}{10^{3} \mathrm{~km} \mathrm{~s}^{-1}}\right)-3.40,
$$

with a correlation coefficient $R=0.80$. BH mass and bulge luminosity are in solar units, and we have excluded PG1704 because of its highly uncertain reverberation BH mass determination (however, if we take the $\mathrm{BH}$ mass and FWHM values quoted in Kaspi et al. (2000) it agrees well with the fit in fig. 4).

The tight correlation (5) is not surprising - it is actually an artifact of the virial relation: since $M_{\mathrm{BH}} \propto v(F W H M)^{2}$, this correlation merely means that $R_{B L R} / L_{\text {bulge }}$ is independent on the line width. Combining eqs. (3) and (5) we have

$$
R_{B L R} \approx 27\left(\frac{L_{\text {bulge }}}{10^{10} L_{\odot}}\right) \quad \text { lt }- \text { days. }
$$

Looking at the data we find almost precisely the same result - a very tight correlation between bulge luminosity and BLR size (in preparation), as shown in fig. 5 .

The best fit to all 55 AGNs in our sample combined is $\log (R /$ lt - days $)=1.05 \log \left(L_{\text {bulge }} / L_{\odot}\right)-8.92$ or

$$
R=13.5 L_{10}^{1.05} \quad \text { lt }- \text { days }
$$

with a correlation coefficient of 0.91 . We note that while Seyferts and NLS1s show a significant correlation, quasars 
alone do not. This is not surprising, as the BLR size of most quasars in our sample is determined from the R$\mathrm{L}$ scaling relation, which is less reliable than reverberation mapping, used for most of the Seyferts. However, the quasars, which do have reverberation measured BLR sizes (solid circles in fig. 5) are indeed very close to the fit.

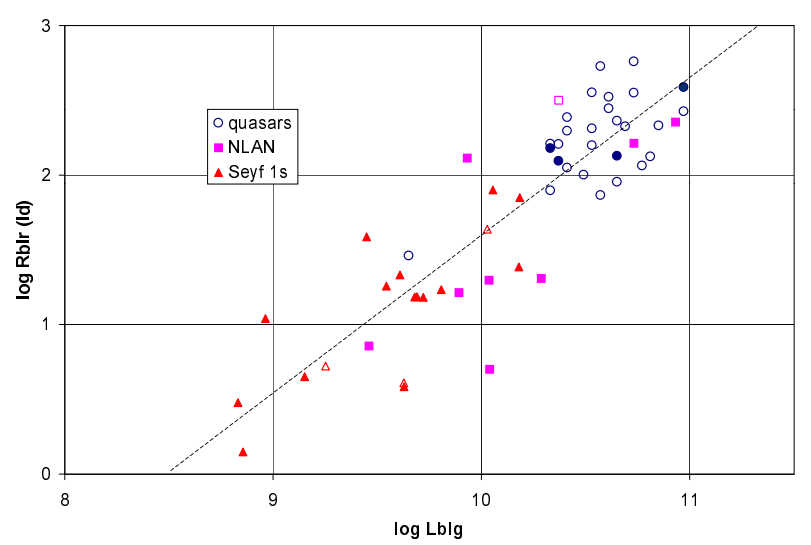

FIG. 5. - The size of the broad emission-line region vs. the bulge luminosity. Red Triangles are Seyfert 1 galaxies, blue circles are quasars and pink squares are narrow line AGNs. Solid symbols indicate $\mathrm{BH}$ masses calculated from reverberation -mapping data (from WPM and Kaspi et al. 2000) and open symbols - calculated from the L-R empirical relation. The solid line indicates the least-squares linear fit.

This is a non-trivial correlation, not just a derivation, as it relates two independent observables: on the one hand the bulge luminosity, a global galactic property on a kpcscale, and on the other hand the BLR size (measured by reverberation mapping or luminosity scaling), on a few light-days scale.

It may be reflecting the $\mathrm{BH}$-bulge relation, but the correlation being much stronger than the $\mathrm{BH}$-bulge relation supports the case that this new relation is more fundamental. A possible connection to basic physical properties may be implied by combining the new BLR-bulge relation, the empirical relation between the BLR size and the centra] source luminosity (e.g. Kaspi et al. 2000) and the BHbulge relation giving that the Eddington ratio increases with $\mathrm{BH}$ mass.

\section{THE BLACK HOLE MASS - BULGE VELOCITY DISPERSION RELATION FOR AGN}

We have shown that the black hole /bulge ratio in NLS1 galaxies seems to be significantly smaller than in broad line AGNs and in quiescent galaxies. However, Seyfert galaxies seem to be consistent with the $\sigma-M_{\mathrm{BH}}$ relation of inactive galaxies (Gebhardt et al. 2000b; Merritt \& Ferrares€ 2001b; Ferrarese et al. 2001). In order to unveil the origin of the lower $\mathrm{BH} /$ bulge ratio of NLANs (namely, whether they really have lower $\mathrm{BH}$ masses or rather larger bulges, relative to the broad line AGN) we look at their location in the $\sigma-M_{\mathrm{BH}}$ plane. The bulge velocity dispersion is measured only for 3 NLS1s (Mrk 110, Mrk 590 and NGC 4051) and 8 broad-line Seyferts (table 1).

We may use the Faber-Jackson relation to estimate the velocity dispersion of other AGNs with measured or es- timated bulge luminosity. Also the relation between the the narrow emission lines and the velocity dispersion (Nelson 2000) may be used to estimate the velocity dispersion in the bulge. Here we use the standard F-J relation, e.g. $L=L_{o} \sigma_{2}^{4}$, where $\sigma_{2}=\sigma / 100 \mathrm{~km} \mathrm{~s}^{-1}$ and $L_{o}$ is a luminosity coefficient determined by a linear fit with a slope of 4 to the observed Seyfert Galaxies (fig. 6).

In our limited data set we note that NLS1s have a significantly larger luminosity coefficient than broad line ones: while for broad line Seyfert 1 galaxies $L_{o}=10^{9} \mathrm{erg} \mathrm{s}^{-1}$, for NLS1s galaxies $L_{o}=6 \times 10^{9} \mathrm{erg} \mathrm{s}^{-1}$. In addition, galaxies with a massive BHs and AGNs in particular, may have a different (flatter) F-J relation than galaxies in general (Wandel 2001 and work in preparation).

Using these relations one may compile a larger sample and increase the dynamical range by including also quasars, to estimate the $\sigma-M_{\mathrm{BH}}$ relation for AGNs. In fig. 7 the $\mathrm{BH}$ mass is plotted against the stellar velocity dispersion. We see that AGNs are consistent with the $M_{\mathrm{BH}}-\sigma$ relation of inactive galaxies $\left(M_{\mathrm{BH}} \propto \sigma^{\alpha}\right.$, with $\alpha=3.5-5$; Gebhardt et al. (2000a) find $\alpha=3.65$ and Merritt and Ferrarese (2001a) give 4.72). Adding the quasars seem to favor a steeper slope, but this result merely reflects the assumed $L \propto \sigma^{4}$ relation; if, for example, a flatter $\sigma-L$ relation is assumed, the luminous objects would have a larger estimated velocity dispersion yielding a flatter slope for the $\sigma-M_{\mathrm{BH}}$ relation. In fig. 7 we indicate by an arrow how the velocity dispersion estimate of luminous objects would move if the best fit to the F-J relation $\left(L \sim \sigma^{3.3}\right)$ for the Seyferts (dotted line in fig 6 ) were used.

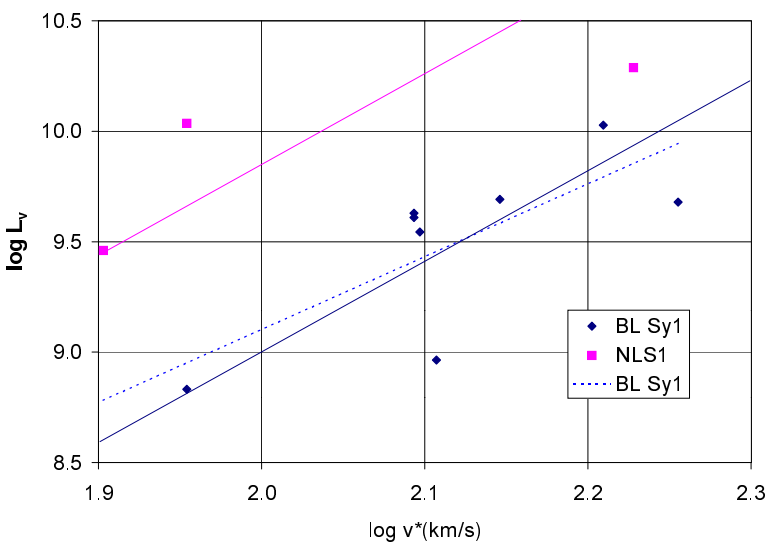

FIG. 6.- Bulge luminosity plotted against stellar velocity dispersion for Seyfert galaxies. Blue Triangles indicate broad-line Seyfert $1 \mathrm{~s}$, pink squares denote NLS1s. The solid lines denote the FaberJackson relation $\left(L \sim \sigma^{4}\right)$ normalized for the broad and narrow line Seyferts, respectively, while the dashed line is the best fit to the broad line Seyferts.

In agreement with previous results (Gebhardt 2000b, Merritt \& Ferrarese 2001b) the NLANs (solid and open pink squares in fig 7) are more or less consistent with the $M_{\mathrm{BH}}-\sigma$ relation, although they do lie at the lower end of the distribution.

The agreement of the $M_{\mathrm{BH}}-\sigma$ relations of inactive galaxies and AGNs (broad and narrow line) suggests that 
the virial masses are essentially correct also for NLANs. The tendency of the NLANs to have low $M_{\mathrm{BH}} / \sigma$ ratios suggests that their lower BH-bulge ratios go along with relatively lower $\mathrm{BH}$ masses than broad line AGNs. In order to settle this issue, more stellar velocity-dispersion measurements of AGNs are required, in particular of NLS1s.

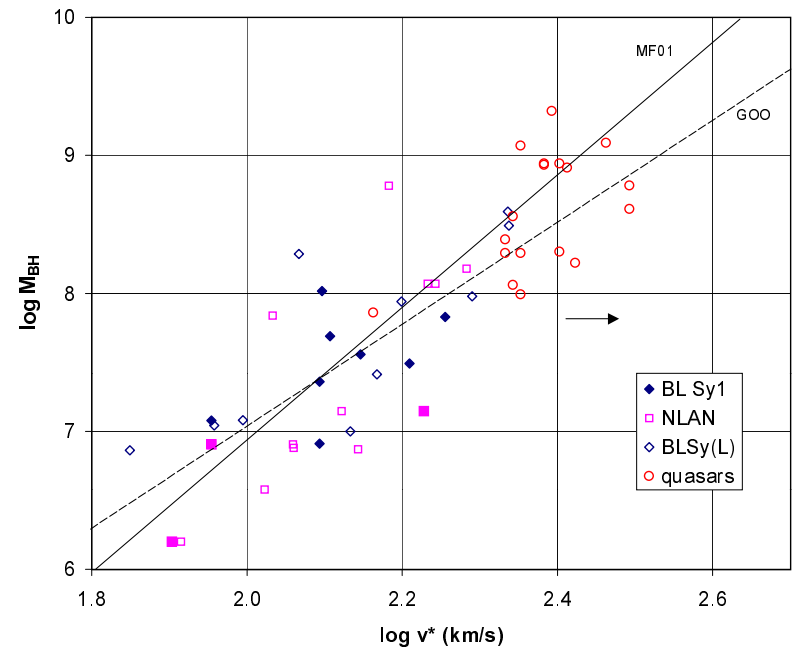

FIG. 7.- Black hole mass of AGNs plotted against the stellar velocity dispersion $\left(v^{*}=\sigma\right)$. Blue triangles are broad line Seyferts, pink squares denote NLS1s and red circles denote quasars. Solid symbols dennote Seyferts with measured $\sigma$, open ones denote Seyferts for which $\sigma$ has been estimated from the Faber-Jackson relation (see text). The dashed and solid lines show the $\sigma-M_{\mathrm{BH}}$ relation of galaxies (Gebhardt et al. 2000a and Ferrarese \& Merritt 2000 , respectively). The arrow shows how the velocity dispersion estimate of luminous objects would typically move if the best fit to the F-J relation for the Seyferts (fig 6) were used.

The difference between NLANs and broad line AGNs in the $\mathrm{BH}$-bulge relation is much larger than the difference in the BH-velocity-dispersion relation. This may be related to the bulge mass (and luminosity) being integral, extended properties, while the stellar velocity dispersion is more closely related to the inner part of the bulge. There is more evidence that the black hole mass in AGN is correlated with the velocity dispersion (and hence the virial mass) on the scale of the central part of the bulge - the narrow line region. This is supported by the correlation between the black hole mass and the velocity dispersion of the narrow emission-line gas, as measured by the [OIII] lines in Seyfert 1 nuclei (Wandel and Mushotzky 1986; Nelson 2000).

\section{DO NLANS REALLY HAVE LOWER BH/BULGE RATIOS?}

We have seen that broad line AGNs follow a similar BHbulge relation as inactive galaxies. The question remains what can be the cause of the lower $\mathrm{BH} /$ bulge ratio in the narrow-line AGNs, and whether this effect is real or apparent.

Several effects have been mentioned that could cause such an apparent lower $\mathrm{BH} /$ bulge ratio (e.g. Wandel 2000): (i) the reverberation - virial method may systematically underestimate the BH mass in NLANs, (ii) the measured bulge luminosity in NLANs may be systematically too large. Alternatively, the difference between the two groups could be real: either (iii) NLANs do have in- trinsically larger (or brighter) bulges, or (iv) they have systematically smaller black holes. In section 5 we have seen that effects (ii) and (iii) are supported by the $\sigma-M_{\mathrm{BH}}$ relation, as the narrow-line Seyferts with measured velocity dispersion (MRK 110, MRK 590 and NGC 4051) seem to have larger bulge luminosity than broad line Seyferts with comparable velocity dispersions (fig. 6). On the other hand, also (i) and (iv) cannot be ruled out, as the reverberation virial BH masses of MRK 590 and NGC 4051 are by a factor 2-3 lower than the average $M_{\mathrm{BH}}-\sigma$ relation of broad line Seyferts and inactive galaxies (fig.7).

There are a number of observational errors that may cause an apparent difference in the black hole - bulge luminosity relation between NLANs and classic AGNs and inactive galaxies, which we discuss below.

\subsection{Errors in estimating bulge luminosity}

As suggested above, the lower $M_{\mathrm{BH}}-L_{\text {bulge }}$ values of NLAN could be accounted for if their bulge luminosity are systematically over estimated. As we saw, this was indeed the case when the Simien-deVaucouleurs method was used for Seyfert galaxies. Could a similar problem exist in the NLAN set? Two of the 3 NLS1s with measured velocity dispersion have low $M_{\mathrm{BH}} / \sigma$ values which may indicate they have relatively small black holes. However, the location of the NLANs in the $M_{\mathrm{BH}}-\sigma$ plot (fig 7) with respect to the inactive and broad line AGNs indicates that the $\mathrm{BH}$ mass can account for only a small part of the difference, suggesting that NLS1s tend to have relatively large bulges. It is however difficult to see why the bulge magnitude of NLANs be systematically over-estimated by a factor of $\sim 10$ (required to explain the difference in the $\mathrm{BH} /$ bulge ratio) compared with the broad line AGNs in the sample.

\subsection{Bias introduced by the stellar kinematics method}

Is it possible that NLANs represent a larger population of galaxies with low black hole mass to bulge luminosity ratios, which is under-represented in the presently available $\mathrm{MBH}$ sample? This could be the case for MBHs detected using stellar dynamics, because this method cannot detect small black holes, and the detection-limit increases with distance. In a resolution-limited method, this would infer a lower detection limit which increases with luminosity. This hypothesis is supported by the distribution of dynamically estimated black hole masses in quiescent galaxies. There are only four inactive galaxies with $\mathrm{BH}$ masses under $10^{7} M_{\odot}$ : the Milky Way, M32 and NGC 7457 detected using stellar dynamics and NGC4945 - with maser dynamics. The two latter galaxies also have low black hole -to bulge luminosity ratios, comparable to the NLAN average ratio. In angular-resolution limited methods, the $\mathrm{MBH}$ detection limit is correlated with bulge luminosity: for more luminous bulges the detection limit is higher, because the stellar velocity dispersion is higher (the Faber-Jackson relation). In order to detect the dynamic effect of a $\mathrm{MBH}$ it is necessary to observe closer to the center, while the most luminous galaxies tend to be at larger distances, so for a given angular resolution the $\mathrm{MBH}$ detection limit is higher. This may imply that the sample is biased towards larger MBHs, as present stellar-dynamical methods are ineffective for detecting MBHs below $\sim 10^{7} M_{\odot}$ (except in 
the nearest galaxies). Since the BLR method is not subject to this constraint, NLANs may represent a low-mass $\mathrm{BH}$ population.

\subsection{The black hole influence on the stellar velocity dispersion}

In section 5 we have seen that the NLANs are not significantly outlying the $\sigma-M_{\mathrm{BH}}$ relation, while they are located far below the $\mathrm{BH}$-bulge relation. In principle this could be caused by the BH influence on the measured velocity dispersion. In NLANs the region influenced by the $\mathrm{BH}$ would be relatively smaller (either because the $\mathrm{BH}$ has a lower mass or because the bulge is larger, and hence has a larger velocity dispersion). This can reduce the difference between narrow and broad line AGNs when $\sigma$ rather than $L_{\text {bulge }}$ is considered. However, as we show below, the $\mathrm{BH}$ influence turns out to be negligible for almost all AGNs in our sample. The effect of the massive $\mathrm{BH}$ on the stellar velocity dispersion can be estimated by comparing the expected velocity field $\left(v \propto\left(M_{\mathrm{BH}} / r\right)^{1 / 2}\right)$ due to the $\mathrm{MBH}$ and the velocity dispersion of the host bulge. The black hole enhances the velocity dispersion in an observed region if its mass is bigger or comparable to the stellar mass within the radius corresponding to the projected angular size. This can be measured by the dimensionless quantity

$$
m=G M_{\mathrm{BH}} / \sigma^{2} \theta D,
$$

where $\sigma$ is the stellar velocity dispersion in the bulge of the host galaxy, $\theta$ is the angular size (e.g. the width of the slit) and $D$ is the distance to the galaxy. In other words, $m$ is roughly the ratio of the $\mathrm{BH}$ mass to the stellar bulge mass inside the radius corresponding to the angular size being sampled. The nuclear velocity dispersion is typically sampled with a slit width of $1-2^{\prime \prime}$ (Ferrarese et al. 2001 use a slit of $\left.2 \times 4^{\prime \prime}\right)$. Since the measured velocity dispersion is weighted by the brightness along the line-ofsight, and since the brightness-density increases steeply at the center, the effective value of $\theta$ is probably smaller than the slit width. The $\mathrm{BH}$ influence parameter can be written as $m=0.9\left(M / 10^{8} M_{\odot}\right) \sigma_{2}^{-2}(D / 10 M p c)^{-1}$, where $\theta$ has been assumed to be $1^{\prime \prime}$. We have calculated $m$ for the 11 Seyferts with velocity dispersion measurements. The three NLS1s have $m \sim 0.01$, lower than the BL Seyferts which have typically $m \sim 0.03-0.1$, with the exception of NGC 3227 which has $m=0.24$. We conclude that the $\mathrm{BH}$ influence on the velocity dispersion in the observed AGNs is negligible.

\subsection{Inclination and BLR geometry}

If the broad emission-line region (BLR) has a flattened geometry and the distribution of inclination angles is biased towards face-on inclinations (as one might expect in the unified model) the velocity inferred from the observed line width may be smaller than the $3 \mathrm{D}$ velocity dispersion, depending on the inclination and the amount of flattening. For example, for a flat BLR configuration viewed at an inclination angle $i$ (where $i=90$ corresponds to face-on), the line-of-sight velocity is smaller than the 3 -D velocity by a factor of $\sin (i)$. The inferred mass would thus be smaller than the actual mass by a factor of $\sin ^{2}(i)$. For a random distribution of inclination angles, the average inferred mass would be decreased by this factor weighted by the distribution, $<\sin ^{2}(i)>=4 \pi \int_{0}^{\pi / 2} \sin ^{2}(i) \cos (i) \mathrm{d} i / 4 \pi=1 / 3$.
Actually, the distribution is probably not random. In the unified scheme Seyfert 1 nuclei are viewed more face-on, within an opening angle of $\lesssim 60$ degrees, so that the weighted average mass-reduction factor is $\left\langle\sin ^{2}(i)\right\rangle=$ $4 \pi \int_{0}^{\pi / 6} \sin ^{2}(i) \cos (i) \mathrm{d} i / 2 \pi=1 / 12$. If the BLR distribution is not flat but with an angular distribution of orbits spanning an angle $\delta$, the effect would be less - between $\sin (i)$ and $\sin (\delta+i)$, depending on the distribution of orbits, so that roughly $\left\langle\sin ^{2}(i+\delta)>\gtrsim 0.1\right.$.

This scenario can explain the lower $M_{\mathrm{BH}} / L_{\text {bulge }}$ values of NLANs. The distribution of inclination angles can also explain the larger scatter of NLANs, compared with broad line Seyferts. In this scenario, NLANs are AGNs with a flattened BLR geometry. seen nearly face-on.

\subsection{Uncertainties and non-virial BLR dynamics}

Is it possible that the black hole masses of NLANs are systematically underestimated by the reverberation - virial method? The reverberation masses of all AGN seem to be more or less correct, in light of the good agreement between AGNs and quiescent galaxies in the case of the black hole - velocity dispersion relation. The uncertainty in the reverberation virial method is not well known. While for individual objects a factor of 2-3 may be representative, the sample average error is probably much smaller. This comes on top of the measurement uncertainty; the uncertainty in the BLR size (calculated from the cross correlation of the continuum and line light curves) can be quite large for objects with poor sampling or low variability. Note that if the virial assumption is incorrect and the gas in the line emitting gas in NLANs is unbound, the gas velocity is actually larger than Keplerian, the black hole mass would have been overestimated, which would increase the BH-bulge discrepancy between NLANs and broad line Seyfert galaxies (and inactive galaxies). Similarly, if the BH mass of broad line AGNs were underestimated, their present good agreement with the BH-bulge relation of quiescent galaxies would deteriorate, as demonstrated in section 3.4 .

More generally, we may ask how reliable are the $\mathrm{BH}$ masses derived by the reverberation virial method? Could there be a systematic effect? The best resolution of HST in relatively nearby galaxies translates into a distance of a few tens of parsecs from the $\mathrm{MBH}$. Reverberation mapping of the broad emission line region in AGNs gives a much closer view - a few light days from the center. Assuming the line-emitting matter is gravitationally bound, having a Keplerian velocity dispersion, it is possible to estimate the virial mass: $M \approx G^{-1} r v^{2}$. This expression may be approximately valid also in the case the line emitting gas is not bound, such as radiation-driven motions and diskwind models (e.g. Murray et al. 1998). The main problem in estimating the virial mass from the emission-line data is to obtain a reliable estimate of the size of the BLR, and to correctly identify the line width with the velocity dispersion in the gas (WPM, Krolik 2001). WPM used the continuum/emission-line cross-correlation function to measure the responsivity-weighted radius $c \tau$ of the BLR (Koratkar \& Gaskell 1991), and the variable (rms) component of the spectrum to measure the velocity dispersion in the same part of the gas, which is used to calculate the 
BLR size, automatically excluding constant features such as narrow emission lines and galactic absorption. Kaspi et al. (2000) find that for most objects in their sample, similar mass estimates are obtained also using either the rms as or the mean FWHM. The case for a central MBH dominating the kinematics in the broad emission-line region is supported by the Keplerian velocity profile $\left(v \propto r^{-1 / 2}\right)$ detected in NGC 5548 (Peterson \& Wandel 1999) and NCG 7469 and 3C390.3 (Peterson \& Wandel 2000). Although different emission-lines have quite different line widths, the delays also vary from line to line in such a way that the virial masses derived from different emission lines are all consistent with a single value. This demonstrates the case for a Keplerian velocity dispersion in the line-width/timedelay data. Another support for the validity of the reverberation virial black hole mass estimates comes from the consistency of the black hole -bulge relations for AGN and inactive galaxies: the $M_{\mathrm{BH}}-\sigma$ relation (Ferrarese et al. 2001), and the $M_{\mathrm{BH}}-L_{\text {bulge }}$ relation (this work).

\subsection{Evolution}

The different BH-bulge relation of NLANs compared with broad-line AGNs and inactive galaxies could suggest that NLANs may be intrinsically different. One possibility is that they are in a different evolutionary stage. Wandel (1999) suggested that in AGNs which are radiating near the Eddington luminosity, the central black hole accretes within a relatively short time scale most of the readily available matter. BHs accreting near the Eddington limit are still growing, hence their mass (relative to the bulge) may be smaller than in AGNs with lower $L / M$ ratios such as Seyferts 1s which may be past the most active phase. Similarly, MBHs in normal galaxies may be the remnants of a past luminous AGN phase, and their BHs did not grow significantly after the decline in the accretion rate. This could explain the discrepancy between narrow and broad line AGNs (NLANs tend to be near the Eddington limit), as well as the similar BH-bulge relations of broad line AGNs and inactive galaxies. This scenario has been followed with a simple model calculation by Wang,
Biermann and Wandel (2000) who examined the relative contribution of mergers to the bulge and $\mathrm{BH}$ mass. For reasonable parameters they find that a $\mathrm{BH} /$ bulge mass ratio of $\sim 0.002$ is obtained by evolution of the $\mathrm{MBH}$ due to Eddington-limited accretion enhanced due to mergers.

\section{SUMMARY}

We re-examine the purported discrepancy between the black hole - bulge luminosity relation of MBHs in nearby galaxies (measured with stellar- and gas dynamics methods) and reverberation -mapped Seyfert nuclei (Wandel 1999). Using updated data. we find that the $\mathrm{BH} /$ bulge relation of AGNs and inactive galaxies are in good agreement over 3 orders of magnitude in bulge luminosity or $\mathrm{BH}$ mass. We show that the apparent difference reported previously originated from overestimated $\mathrm{BH}$ masses in quiescent galaxies, overestimated bulge luminosity for the Seyferts and including narrow-line Seyfert 1s which seem to have genuinely low $\mathrm{BH} /$ bulge ratios. We find that the lower $\mathrm{BH} /$ bulge ratio of narrow line AGNs is part of a more general inverse correlation of the $\mathrm{BH} /$ bulge ratio with the emission-line width. Considering the $\mathrm{BH}$-velocity dispersion correlation, the lower $\mathrm{BH} /$ bulge ratio of narrow-line AGNs seems to be related (at least in part) to the host, as narrow and broad line AGNs (as well as inactive galaxies) seem to have a similar $\sigma-M_{\mathrm{BH}}$ relation. We predict the velocity dispersion of high luminosity AGN using the Faber Jackson relation derived from Seyfert galaxies for with a measured $\sigma$. We discuss three classes of explanations for the lower $\mathrm{BH} /$ bulge ratios of narrow-line AGNs: black hole mass - bulge luminosity relation of Seyfert galaxies and MBHs in normal galaxies: Observational or methodrelated errors or bias, intrinsic and orientation-related effects and Evolution.

I acknowledge support from BSF grant 1999-336 and the hospitality of the astronomy department at UCLA, as well as helpful discussions with Laura Ferrarese, Karl Gebhardt, Luis Ho, Ari Laor and David Merritt.

\section{REFERENCES}

Bahcall, J.N., Kirhakos,S.,Saxe,D.H. \& Schneider, D.P. 1997, ApJ, 479, 642 Princeton U. 1987

Cranshaw, D.M. 1986, ApJS62, 861.

Erackleous, M. and Halpern, J.P. 1994 ApJS, 90, 1

Ferrarese, L. and Merritt, D. 2000 ApJ, 539, L9

Ferrarese, L., Pogge, R.W., Peterson, B.M. ,Merritt, D. , Wandel, A. \& Joseph, C.L. 2001, ApJ, 555, L79

Gebhardt, K. et al. 2000a ApJ, 539 L13

- 2000b ApJ, 543, L5

Hamilton, T.S., Casertano, S. \& Turnshek,D.A. 2001, ApJ, in press (astro-ph 0011255)

Ho, L.C. 1999 in Observational Evidence for Black Holes in the Universe, ed. S.K.Chakrabatri (Dordrecht: Kluwer) 153

Kaspi, S. et al. 2000, ApJ, 533, 631

Kirhakos, S., Bahcall, J.N., Schneider, D.P. \& Kristian, J. 1999, ApJ, 520, 67

Koratkar, A.P., \& Gaskell, C.M. 1991, ApJS, 75, 719

Kormendy, J., \& Richstone, D. 1995, ARA\&A, 33, 581

Kormendy, J. \& Gebhardt, K. 2001, proc. of the 20th

Texas Symposium, eds. H.Martel \& J.C.Wheeler, in press (astro-ph/0105230)

Krolik, J.H. 2001, ApJ, 551, 72.

Laor, A. 1998, ApJ, 505, L83

Laor, A. 2001, ApJ, 553, 677

Magorrian, J. et al. 1998, AJ, 115, 2285
Mathur, S.,Kuraszkiewicz, J. \& Czerney, B. 2001, New Astronomy, in press (astro-ph/0102356)

McLure, R.J. \& Dunlop, J.S 2001, MNRAS, in press (astroph/0009406, MD01)

Merritt, D. and Ferrarese, L. 2001a, MNRAS, 320, L30

- 2001b ApJ, 547, 140

Murray, N. 1998, ApJ, 494, 125

Nelson, C.H. \& Whittle, M. 1995 ApJS, 9967

Nelson, C.H. 2000, ApJ, 544, L91

Neugebauer, G. et al. 1987, ApJS, 63, 615

Peterson, B. \& Wandel, A. 1999, ApJ, 521 L95

- 2000 ApJ, 540, L13

Richstone, D. et al. 1998, Nature, 395, A14

Simien, F. \& de Vaucouleurs, G 1986, ApJ, 302, 564

Santos Lleo, M., Clavel J., Schulz, B. et al. 2001 Astronomy and Astrophysicsin press (astro-ph0102356)

Virani, S.N., De Robertis, M.M. and Van Delfsen, M.L. 2000, AJ120, 1739.

Wandel, A. 1999, ApJ, 519, L39

- 2000, in "Probing the Physics of AGN by Multiwavelength Monitoring", ASP 224, 365, eds. B.M. Peterson,P.S.Polidan \& R.W.Pogge - 2001, BAAS, 33, 898.

Wandel, A. \& Mushotzky, R.F. 1986, ApJ, 306, L61

Wandel, A., Peterson, B. and Malkan, M 1999 (WPM), ApJ, 526, 579 
Whittle, M. et al. 1992, ApJS, 79, 49

Wang, Y.P., Biermann, P. \& Wandel, A. 2000, Astronomy and Astrophysics, 361,550 